\title{
Characterizing the coverage of critical effects relevant in the safety evaluation of food additives by AOPs
}

\author{
Nynke I. Kramer ${ }^{1} \cdot$ Yvette Hoffmans $^{2} \cdot$ Siyao Wu $\mathrm{Wu}^{2}$. Anette Thiel ${ }^{3} \cdot$ Natalie Thatcher $^{4}$ - Timothy E. H. Allen ${ }^{5}$. \\ Sara Levorato ${ }^{6} \cdot$ Heinz Traussnig $^{7} \cdot$ Stefan Schulte $^{8} \cdot$ Alan Boobis $^{9} \cdot$ Ivonne M. C. M. Rietjens $^{10} \cdot$ Mathieu Vinken $^{11}$
}

Received: 16 April 2019 / Accepted: 17 June 2019 / Published online: 29 June 2019

(c) The Author(s) 2019

\begin{abstract}
There is considerable interest in adverse outcome pathways (AOPs) as a means of organizing biological and toxicological information to assist in data interpretation and method development. While several chemical sectors have shown considerable progress in applying this approach, this has not been the case in the food sector. In the present study, safety evaluation reports of food additives listed in Annex II of Regulation (EC) No 1333/2008 of the European Union were screened to qualitatively and quantitatively characterize toxicity induced in laboratory animals. The resulting database was used to identify the criti$\mathrm{cal}$ adverse effects used for risk assessment and to investigate whether food additives share common AOPs. Analysis of the database revealed that often such scrutiny of AOPs was not possible or necessary. For $69 \%$ of the food additives, the report did not document any adverse effects in studies based on which the safety evaluation was performed. For the remaining $31 \%$ of the 326 investigated food additives, critical adverse effects and related points of departure for establishing health-based guidance values could be identified. These mainly involved effects on the liver, kidney, cardiovascular system, lymphatic system, central nervous system and reproductive system. AOPs are available for many of these apical endpoints, albeit to different degrees of maturity. For other adverse outcomes pertinent to food additives, including gastrointestinal irritation and corrosion, AOPs are lacking. Efforts should focus on developing AOPs for these particular endpoints.
\end{abstract}

Keywords Food additives · Critical adverse effect · Adverse outcome pathway · Acceptable daily intake · 3Rs

\section{Abbreviations \\ 3Rs Replacement, refinement, and reduction of animal tests \\ ADI Acceptable daily intake}

Electronic supplementary material The online version of this article (https://doi.org/10.1007/s00204-019-02501-x) contains supplementary material, which is available to authorized users.

Mathieu Vinken

mathieu.vinken@vub.be; publications@ilsieurope.be

1 Institute for Risk Assessment Sciences, Utrecht University, PO Box 80177, 3508 TD Utrecht, The Netherlands

2 Wageningen University and Research, Droevendaalsesteeg 4, 6708 PB Wageningen, The Netherlands

3 DSM Nutritional Products Ltd., Wurmisweg 576, 4303 Kaiseraugst, Switzerland

4 Mondelēz International, Bournville Place, Bournville Ln, Birmingham B30 2LU, UK

5 Department of Chemistry, Centre for Molecular Informatics, University of Cambridge, Lensfield Road, Cambridge CB2 1EW, UK
AOP(s) Adverse outcome pathway(s)

BMDL Benchmark dose lower confidence limit

CAS Chemical abstracts service

EDI Estimated daily intake

EFSA European Food Safety Authority

EU European Union

ILSI International Life Sciences Institute

6 Unilever, Safety and Environmental Assurance Centre (SEAC), Sharnbrook, Bedford MK441LQ, UK

7 Mayr-Melnhof Karton Gesellschaft M.B.H., Frohnleiten Mill, Wannersdorf 80, 8130 Frohnleiten, Austria

8 Department of Product Safety, BASF SE, 67056 Ludwigshafen, Germany

9 Department of Medicine, Imperial College London, London W12 0NN, UK

10 Division of Toxicology, Wageningen University, Stippeneng 4, 6708 WE Wageningen, The Netherlands

11 Department of In Vitro Toxicology and Dermato-Cosmetology, Vrije Universiteit Brussel, Laarbeeklaan 103, 1090 Brussels, Belgium 


\begin{tabular}{|c|c|}
\hline IPCS & International Program on Chemical Safety \\
\hline JECFA & $\begin{array}{l}\text { Joint FAO/WHO Expert Committee on Food } \\
\text { Additives }\end{array}$ \\
\hline $\mathrm{KE}(\mathrm{s})$ & Key event (s) \\
\hline LOAEL & Lowest observed adverse effect level \\
\hline MIE & Molecular initiating event \\
\hline $\operatorname{MoS}$ & Margin of safety \\
\hline OECD & $\begin{array}{l}\text { Organization for Economic Cooperation and } \\
\text { Development }\end{array}$ \\
\hline $\mathrm{NO}(\mathrm{A}) \mathrm{EL}$ & No observed (adverse) effect level \\
\hline PoD & Point of departure \\
\hline SCF & Scientific Committee on Food \\
\hline WHO & World Health Organization \\
\hline
\end{tabular}

\section{Introduction}

Historically, the safety of food additives has been assessed according to the risk assessment paradigm described by the World Health Organization (WHO) (WHO 2009). Safety evaluation of food additives consists of four steps: (1) hazard identification, i.e., the assessment of the intrinsic toxic properties of the compound; (2) hazard characterization, which includes dose-response assessment, identification of critical toxic endpoints and derivation of health-based guidance values; (3) exposure assessment, i.e., the estimation of exposure/intake; (4) risk characterization, which integrates the information from the previous steps to evaluate the risk associated with the exposure to the food additive. Within hazard characterization, an acceptable daily intake (ADI) value is established, which is defined as the maximum intake level that poses no harm to the consumer upon daily exposure over an entire lifetime. Thus, to conclude on safe use, the estimated daily intake (EDI) of the consumer resulting from the use of a food additive should not exceed the ADI (i.e., risk characterization). For hazard identification and characterization, several endpoints need to be assessed (WHO 2009). The core endpoints are mutagenicity/genotoxicity, reproductive toxicity and developmental toxicity, and repeated dose toxicity, including carcinogenicity. Evaluation of some of these endpoints, in particular mutagenicity/genotoxicity, is predominantly based on in vitro tests. However, the evaluation of systemic endpoints generally relies on toxicity studies in laboratory animals. This safety assessment paradigm is used worldwide by (inter)national regulatory authorities, not only for food additives, but also for industrial chemicals, plant protection products, biocides and (veterinary) pharmaceuticals (Embry et al. 2014).

In the European Union, approved food additives are listed in Annex II of Regulation (EC) No 1333/2008 (EC1333/2008). All the authorized food additives are deemed safe. Indeed, according to the Regulation, "A food additive may be included in the Community lists in Annexes
II and III only if it meets the following conditions [...] (a) it does not, on the basis of the scientific evidence available, pose a safety concern to the health of the consumer at the level of use proposed" (EC 1333/2008). In addition, a reevaluation of food additives that were already permitted in the European Union before 20 January 2009 has been set up under Regulation (EU) No 257/2010 and is still ongoing. The European Food Safety Authority (EFSA), and previously the Scientific Committee on Food (SCF), are responsible for the safety assessment as mandated by the European Commission. An ADI is usually derived when adequate information on toxicity is available. Nevertheless, some food additives have no ADI due to low intrinsic toxicity. In this case, there is no need for a numerical ADI, as there is no safety concern at the reported uses and use levels (EFSA 2014a). On the other hand, when the toxicity database is not adequate to establish an ADI, the safety of the food additive is assured by calculating the margin of safety (MoS), i.e., comparing the highest estimated intake with the lowest point of departure (PoD) available. For re-evaluation purposes and new approvals, EFSA published guidance documents and, if needed, a call for data. The safety assessment requires the evaluation of data on toxicokinetics, genotoxicity, subchronic/chronic toxicity, and carcinogenicity (EFSA 2012). Improvements have been achieved in terms of a more flexible and tailor-made testing approach. However, hazard identification and characterization for systemic endpoints, such as target organ-specific toxicity, reproductive toxicity, and developmental toxicity, still rely on animal experimentation, which, in addition to obvious ethical concerns, can lack human relevance (Meigs et al. 2018).

Given the ethical, financial and scientific concerns associated with traditional animal testing in safety assessment of food additives, there is a push towards incorporating more non-animal approaches (Blaauboer et al. 2016; EFSA 2009, 2014b; NRC 2007). In this respect, a roadmap has been proposed in which the focus moves away from the "black box" approach inherent to animal toxicity testing towards one where understanding the molecular mechanisms of toxicity becomes central (Blaauboer et al. 2016). The roadmap formally incorporates knowledge about the progression of toxicity events across scales of biological organization that lead to adverse outcomes in humans, generally referred to as the adverse outcome pathway (AOP) concept. AOPs are pragmatic tools to rationally and visually capture existing knowledge regarding the mechanistic basis of toxicity. An AOP starts from a molecular initiating event (MIE) (i.e., a trigger of toxicity) and relies on a series of key events (KEs), linked by key event relationships, ultimately resulting in a specific toxicological effect (i.e., the adverse outcome) (Ankley et al. 2010). AOPs are chemical-agnostic in that they describe a toxicological process from a purely dynamic perspective (Becker et al. 2015; Burden et al. 2015; 
Edwards et al. 2016; Perkins et al. 2015; Villeneuve et al. 2014). Information from different sources can be used during AOP development, including in silico, in vitro, in vivo and epidemiological data (Vinken et al. 2017). AOP development ideally complies with guidance from the Organization for Economic Cooperation and Development (OECD) (OECD 2017). In fact, the OECD, in collaboration with a number of other organizations, has established an electronic repository for AOPs, called the AOP Wiki. At present, the AOP Wiki contains about 280 AOPs for a plethora of toxicological effects (http://aopkb.org). AOPs were intended particularly to support regulatory decision-making based on the desire to make effective use of mechanistic data, particularly novel data streams that can be generated more rapidly and cost-effectively in a high-throughput format, rather than relying only on apical outcome data traditionally measured in whole-organism toxicity tests (Burden et al. 2015; Dean et al. 2017; Delrue et al. 2016; Edwards et al. 2016; Villeneuve et al. 2014). The specific application of an AOP is, however, usually dictated by the amount of available experimental and observational data as well as the AOP's degree of maturity (Leist et al. 2017; Vinken et al. 2017).

In the present paper, a recent study performed by the expert group on "Adverse outcome pathways and risk assessment" within the task force of "New approaches to chemical risk assessment for foods and food ingredients" at the International Life Sciences Institute (ILSI) Europe is described. Safety evaluation reports of all food additives listed in Annex II of Regulation (EC) No 1333/2008 were screened to qualitatively and quantitatively characterize the critical adverse effects induced by these food additives in laboratory animals. Subsequently, possible adverse outcomes were benchmarked against available AOPs in the AOP Wiki. The goal of this study was threefold, namely (1) to collect the ADIs of food additives, (2) to identify the critical adverse effects used for the safety assessment, and (3) to investigate whether food additives share common AOPs. The outcome of this study will shed light onto the potential application of AOPs for the safety evaluation of food additives.

\section{Materials and methods}

All food additives authorized in the EU according to Regulation (EC) No 1333/2008 and successive amendments are included in the present analysis. The data were retrieved from safety assessment reports published by regulatory authorities [i.e., EFSA/SCF or the Joint FAO/WHO Expert Committee on Food Additives (JECFA)] and were listed in a Microsoft Excel ${ }^{\circledR}$ spreadsheet (Table S1; supplementary information). The cutoff date for the data included in this study is October 2018. Table S1 includes, for all food additives, E-number, name, chemical abstracts service (CAS) number, functional class, ADI (when defined), PoD from which the ADI was derived, and adverse outcome used to define this PoD. Functional classes of listed food additives include food colorings (E 100-181), preservatives (E 200-290), acidity regulators and antioxidants (E 300-385), emulsifiers and stabilizers (E 400-495), anti-caking agents (E 500-578), flavor enhancers (E 620-641), glazing agents and others (E 900 and onwards). Likewise, the type of animal study, species and reference of the study were recorded. EFSA evaluations were used for data collation where possible. When not available, the evaluations of the SCF or JECFA were consulted. For the purpose of this study, the adverse outcome on which a current ADI is based was considered the most relevant and data entry was restricted to these critical adverse outcomes.

During the retrieval of the ADIs, different ADI designations were encountered: (1) EFSA/SCF established a numerical ADI, sometimes a group ADI for food additives sharing similar structures or common metabolic pathways; (2) no ADI was specified, since the MoS for the ADI resulting from the proposed use was considered sufficiently large; (3) no ADI was specified, since the compound had been tested and was not toxic at the highest dose tested. In Table S1 (supplementary information), a color coding was introduced to discriminate between the different types of adverse outcomes in toxicity studies used for the safety assessment. The classes were defined as: (1) "no adverse effect at highest dose level" (blue); (2) "local toxicity" (green); (3) "non-specific toxicity" (red); (4) "specific systemic toxicity" (orange). The blue code ("no adverse effect at highest dose level") was used when the no observed (adverse) effect level (NO(A)EL) of the food additive was the highest dose level tested in the animal studies. In the vast majority of these cases, the NOAEL was above or close to the limit dose of $1000 \mathrm{mg} / \mathrm{kg}$ body weight/day. The green code ("local toxicity") was applied to effects, such as local irritation of the stomach or other parts of the gastrointestinal tract, as well as for effects like laxation and loose stool, where it was assumed and likely that the observation is due to a local effect. The red code ("nonspecific toxicity") was used for adverse effects for which no organ-specific toxicity or any specific mode-of-action could be identified, such as reduced body weights, body weight gain, reduced food consumption and lower litter size. The orange code ("specific systemic toxicity") was used when adverse effects were observed in a specific organ or system. A grey color was used in situations where no numerical ADI was set because no adverse effects were reported in toxicological studies and the current exposure does not raise a safety concern.

The aforementioned classification of chemicals by adverse effects allowed for a general analysis of common types of critical endpoints used for the safety evaluation of food additives, functional additive classes and toxic 
potencies. The identified adverse outcomes were benchmarked against available AOPs in the AOP Wiki (http:// aopkb.org/) in October 2018. More specifically, AOPs applicable to rodents and, if available, humans, were identified by searching for particular key words in AOP titles and abstracts, such as "epithelial hyperplasia", "fetal weight" and "hepatotoxicity". Since AOPs in the AOP Wiki significantly differ in degree of maturity, only those that were sufficiently developed and documented (i.e., those with defined MIE, KEs and adverse outcome) were considered in this study.

\section{Results}

\section{Overview of critical toxic endpoints used for the safety assessment of food additives currently authorized in the EU}

Table S1 (supplementary information) summarizes the toxicity data collected from safety evaluations of food additives and forms the basis of this study. The resulting database contains 326 food additives of which the majority are emulsifiers and stabilizers (87), acidity regulators and antioxidants (58) (Fig. 1a). For 35\%-70\% of the food additives in each category, no specific toxicity study was available to establish a chemical-specific ADI and/or to assign an adverse outcome. Of the 133 chemicals for which a PoD was available, all but six additives had NOAELs as a PoD. For the remaining additives, the LOAEL or benchmark dose lower confidence limit (BMDL) values were used to derive an ADI. Of the PoD values, ammonia caramel (E 150c) had the highest $\mathrm{PoD}(20,000 \mathrm{mg} / \mathrm{kg}$ body weight/day in rats) and the ferrocyanides (E 535-538) had the lowest PoD (4.4 mg/kg body weight/day in rats). Food additives in the acidity regulators and antioxidants, colorings, preservatives and anti-caking agents functional classes were found to have the lowest PoDs, although the range in PoD values varied by up to four orders of magnitude (Fig. 1b). Of the identified PoD values below $50 \mathrm{mg} / \mathrm{kg}$ body weight/day, the majority were for metal-containing compounds and the predominant adverse effects were developmental and neurodevelopmental toxicity. These effects included preweaning mortality and postnatal growth retardation in a two-generation reproduction and developmental toxicity study for hexamethylene tetramine (E 239) and neurodevelopmental effects in offspring of mice in a two-generational reproductive toxicity study for aluminium sulphate (E 520). Moreover, some food additives were found to be nephrotoxic, including sodium, potassium and calcium ferrocyanide (E 535, E 536, and E 538).

As with PoDs, less than half of the food additives have a numerical ADI. In fact, 196 of the 326 food additives (i.e., 60\%) do not have a numerical ADI. The high number of food additives without an ADI assigned is due to several reasons. This includes the fact that the available
A

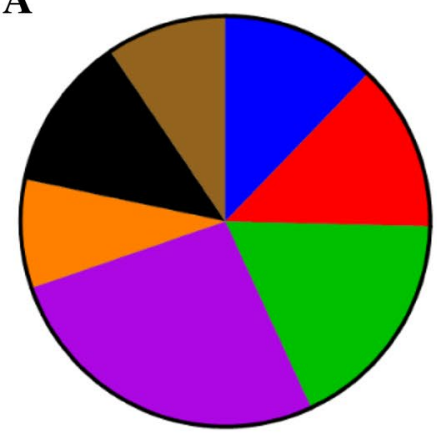

colorings

- preservatives

- acidity regulators and antioxidants

- emulsifiers and stabilizers

anti-caking agents

- flavor enhancers

glazing agents and others
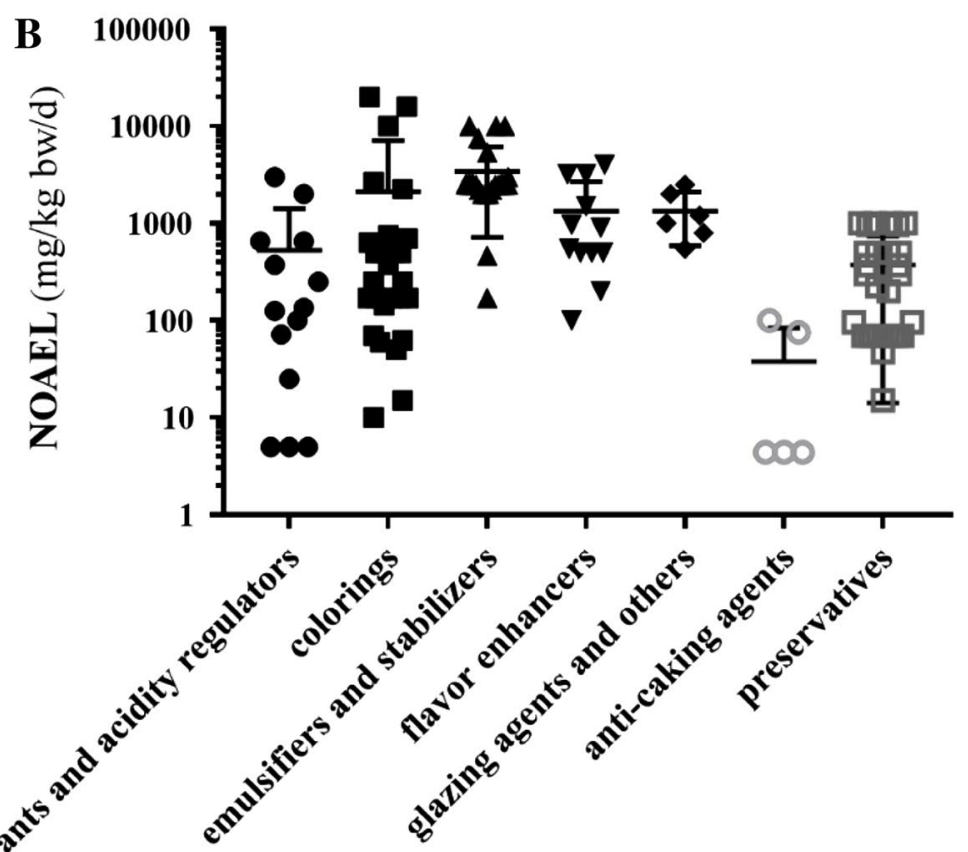

\section{functional classes}

Fig. 1 Classification of 326 food additives into functional classes (a) and associated NOAEL for setting the ADI (b), where available and in comparable units $(\mathrm{mg} / \mathrm{kg}$ body weight/day) 
toxicological data are insufficient to establish an ADI, while the level of exposure to the food additive is not of safety concern when considering the current uses and use levels. This is the case for thermally oxidized soya bean oil interacted with mono- and diglycerides of fatty acids (E 479b) and iron tartrate (E 534), for which EFSA concluded that the MoS calculated comparing the highest estimated exposure and the lowest available NOAEL is sufficiently high, and therefore there is no safety concern for the use and use level proposed (EFSA 2015, 2018). It should be noted that despite not having an ADI, the adverse effects observed in toxicity studies with these food additives were still considered for possible association with an existing AOP. For the vast majority of food additives, an ADI is not deemed necessary because no toxic effects were seen at the highest tested dose in animal studies and the exposure data indicate that there is no safety concern at the currently reported uses and use levels. Some of these food additives are authorized at quantum satis in specified food categories, which means that no maximum level is specified by the Regulation. This situation occurs for ascorbic acid and related salts (E 300-302), salts of lactate (E 325-327), salts of malate (E 350-352) or citric acid and related salts (E 331-333). This lack of toxicity even at high dose levels implies there is no concern over use of such compounds as food additives, but also that in most cases, the available data do not provide critical adverse effects that could be subject to AOP analysis. It is therefore not surprising that $60 \%$ of the food additives analyzed in this study do not have an ADI established and this confirms that, in contrast to pesticides for example, food additives are not expected to have toxicity.

The remaining $40 \%$ of food additives presented in Table S1 (supplementary information) do have a numerical ADI, but do not necessarily have specific toxicities associated with them. The first of three categories of food additives with ADIs comprises of food additives for which the numerical ADI is based on a NOAEL that is the highest dose level tested. For these chemicals, no adverse effects are observed at the highest dose level tested, which is used as the PoD to establish the numerical ADI. This situation is observed, for instance, for polyglycerol polyricinoleate (E 476). The ADI of $25 \mathrm{mg} / \mathrm{kg}$ body weight/day is based on the only dose level tested of $2500 \mathrm{mg} / \mathrm{kg}$ body weight/day in a 2-year combined chronic toxicity/carcinogenicity study in rats that did not reveal adverse effects (EFSA 2017). Logically, for these chemicals, the PoD does not provide input for evaluating the connection with existing AOPs.

The second group of food additives with a numerical ADI includes those for which the ADI is based on a non-specific toxic endpoint, such as weight loss, soft stool or diarrhea. For example, a reduction in body weight gain is observed in rats exposed to sucralose (E 955) and sucrose esters of fatty acids (E 473). These types of adverse effects are considered not sufficiently specific to relate to an existing AOP.

The third group comprises food additives that have a numerical ADI that is based on a specific adverse effect, such as hepatotoxicity, nephrotoxicity, reproductive toxicity, carcinogenicity or immunotoxicity. These food additives provide useful input to analyze the potential of linkage of the adverse effects with existing AOPs, and therefore illustrate the possible value of AOPs in the safety evaluation of food additives. This third group comprises 71 out of the 326 food additives analyzed (22\%). Figure 2 summarizes the categories of adverse effects associated with food additive exposure in toxicity studies.

\section{Food additives associated with specific toxicity}

An analysis of the types of adverse outcomes associated with food additive exposure was carried out using the data for the 71 food additives for which a specific adverse effect was described, 14 of which had no established ADI. The adverse outcomes of the 71 food additives were related to various organs and systems, including the liver, kidney and cardiovascular system, as well as developmental and reproductive processes and the endocrine system. Effects on the liver, the kidneys, the cardiovascular system, lymphatic circulation and developmental processes/reproductive system are listed in Tables 1, 2, 3, and 4, respectively.

In addition, there were effects on hormonal systems, like on the thyroid for butylated hydroxytoluene (E 321). For several food additives, local irritation and/or corrosivity or diarrhea and/or vomiting were reported, which, depending on the underlying mode-of-action, may be related to nonspecific effects or present, for example, possible immunotoxicity or gastrointestinal toxicity. Only in two cases, the critical toxic endpoint was carcinogenesis, namely for dimethyl dicarbonate (E 242) and saccharins (E 954). Increased incidence in hepatocellular adenomas and carcinomas has been reported in only one sex of one species of animals (i.e., female F344 rats) exposed to one of the reaction products of dimethyl dicarbonate (E 242), namely methyl carbonate.

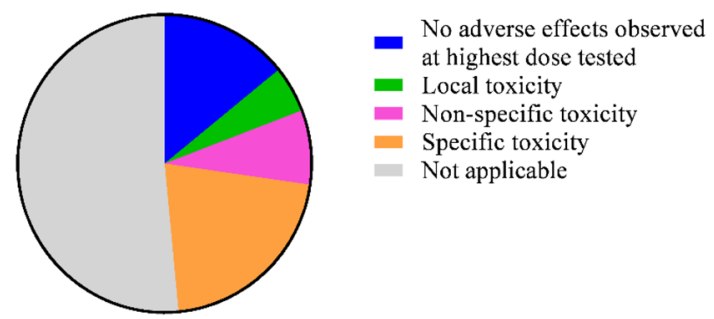

Fig. 2 The type of toxicity associated with exposure to each of the 326 food additives has been categorized into five categories and summarized in a pie chart. "Not applicable" refers to food additives for which an ADI is not deemed necessary 
Table 1 Effects of food additives on the liver

\begin{tabular}{ll}
\hline Effect & Food additive \\
\hline $\begin{array}{l}\text { Changes in clinical chemistry (increases in alanine aminotrans- } \\
\text { ferase or alkaline phosphatase levels) }\end{array}$ & Lycopene (E 160d), iron tartrate (E 534), neotame (E 961), oxidized polyethyl- \\
Increased serum bile acid levels & ene wax (E 914), annatto, bixin, norbixin (E 160b) \\
Hepatocellular hypertrophy & Aron tartrate (E 534) \\
Hepatocellular vacuolation & Annatto, bixin, norbixin (E 160b) \\
Focal liver necrosis & Paprika extract, capsanthin, capsorubin (E 160c), microcrystalline wax (E 905) \\
Leucocyte infiltration & Annatto B (E 160b), hydrogenated poly-1-decene (E 907) \\
Hepatotoxicity & Brown HT (E 155) \\
Fatty liver & Alpha-tocopherol (E 307) \\
\hline
\end{tabular}

Table 2 Effects of food additives on the kidneys

\begin{tabular}{|c|c|}
\hline Effect & Food additive \\
\hline Altered kidney weight & $\begin{array}{l}\text { Sorbitan monopalmitate (E 495), iron tartrate (E 534), annatto, bixin, norbixin (E 160b), steviol glycosides (E 960), } \\
\text { litholrubine BK (E 180) }\end{array}$ \\
\hline Increased excretion rate & Sodium ferrocyanide (E 535), potassium ferrocyanide (E 536) \\
\hline $\begin{array}{l}\text { Accumulation of eosino- } \\
\text { philic material }\end{array}$ & Beta-apo-8'-carotenal (E 160e) \\
\hline Altered urinalysis & Annatto, bixin, norbixin (E 160b), beta cyclodextrin (E 459) \\
\hline Nephrocalcinosis & $\begin{array}{l}\text { Phosphoric acid (E 338), sodium phosphates (E 339), potassium phosphates (E 340), calcium phosphates (E 341), } \\
\text { magnesium phosphates (E 343) }\end{array}$ \\
\hline Nephrosis & $\begin{array}{l}\text { Sorbitan monopalmitate (E 495), sorbitan monostearate (E 491), sorbitan tristearate (E 492), sorbitan monolaurate (E } \\
\text { 493), sorbitan monooleate (E 494), sorbitan monopalmitate (E 495), ponceau 4R, cochineal red A (E 124) }\end{array}$ \\
\hline
\end{tabular}

Table 3 Effects of food additives on the cardiovascular system or lymphatic circulation

\begin{tabular}{ll}
\hline Effect & Food additive \\
\hline Altered heart weight & Brown HT (E 155) \\
Arrhythmia & Calcium sulphate (E 516), calcium chloride (E 509) \\
Hematological changes & Propyl gallate (E 310), tertiary-butyl hydroquinone (E 319), butylated hydroxytoluene (E 321), hydro- \\
& genated poly-1-decene (E 907), propane-1,2-diol (E 1520) \\
Lowered hemoglobin levels & Azorubine and stannous chloride (E 512), methemoglobin formation reported for potassium nitrite (E \\
Changes in leucocyte/neutrophil counts & 249), sodium nitrite (E 250), sodium nitrate (E 251), potassium nitrate (E 252) \\
& Sorbitan monopalmitate (E 495), iron tartrate (E 534), ethyl lauroyl arginate (E 243), brown HT (E \\
\hline
\end{tabular}

Table 4 Effects of food additives on developmental processes and the reproductive system

\begin{tabular}{ll}
\hline Effect & Food additive \\
\hline Testicular toxicity & Cyclamates (E 952) \\
Retarded growth of the offspring & Hexamethylene tetramine (E 239), butylated hydroxytoluene (E 321) \\
Cystic ovaries & Brown HT (E 155) \\
Altered ovary weight & Steviol glycosides (E 960) \\
General reproductive and devel- & $\begin{array}{c}\text { Butylated hydroxytoluene (E 321) and neurodevelopmental changes } \\
\text { opmental effects }\end{array}$ \\
& reported for aluminium (E 173), aluminium salts (E 520-523), gluta- \\
& mates (E 620-625) \\
\hline
\end{tabular}


EFSA noted that there was no genotoxicity concern for methyl carbonate and that the exposure remains below the threshold of toxicological concern of $30 \mu \mathrm{g} / \mathrm{kg}$ body weight/ day for Cramer class I substances. This means that there is no indication for a safety concern for the use of dimethyl dicarbonate (E 242) as a food additive at its currently reported uses and use levels. Saccharin (E 954) has been shown to be a non-genotoxic carcinogen at only one site in only one sex of one species of animals (i.e., bladder of male rats). Considering the weight-of-evidence, the SCF stated that it is unlikely that the tumors in the male rat bladder are of relevance for humans. However, the SCF, as a matter of prudence, decided to take these lesions into account in setting an ADI for saccharins (E 954).

\section{Screening of the AOP Wiki for AOPs relevant to food additives}

The AOP Wiki contains about 280 AOPs for a broad variety of adverse effects in human toxicology and ecotoxicology, whereby both general and organ-specific toxicities are considered (http://aopkb.org/). The toxicological endpoints evaluated in animal studies and used in the safety evaluation of food additives can be easily related to the adverse outcome described in an AOP, albeit to a diverging extent of detail or completeness. On the other hand, no information could be found on the MIEs in the safety evaluation reports of the food additives included in this study. Therefore, it is not possible to unequivocally link existing AOPs to adversity induced by food additives in laboratory animals. Nevertheless, several available AOPs link to specific toxicological endpoints identified in this study (Table 5). Numerous AOPs relate to toxicity in liver and kidney, which have been identified as potential target organs for some food additives. Annatto B (E 160b), lycopene (E 160d) and iron tartrate (E 534) increase levels of aminotransferases, which is a general marker of liver toxicity, or cause undefined hepatotoxicity, as holds for alpha-tocopherol (E 307). Liver steatosis can be triggered by various MIEs, all of which have been embedded in different AOPs (AOP34/36/57/58/59/60/61/62/232) and was found to be induced by oxidized polyethylene wax (E 914). Iron tartrate (E 534), oxidized polyethylene wax (E 914) and neotame (E 961) increases levels of bile acids and/or alkaline phosphatase, which are clinical indicators of cholestasis, for which an AOP (AOP27) is available.

For kidney, specific toxic manifestations are less delineated compared to liver, both in the EFSA/SCF/JECFA safety evaluation reports of the food additives as well as in the AOPs in the AOP Wiki. In fact, there are several AOPs describing nephrotoxicity (AOP33/105/116/128/138 /177/186/256/257/258), all developed to a different degree of maturity. As is the case for hepatic adversities, none of these kidney-related AOPs are unambiguously applicable to food additives for which various signs of renal toxicity have been reported, such as increased excretion rate [sodium ferrocyanide (E 535), potassium ferrocyanide (E 536) and calcium ferrocyanide (E 538)], altered urinalysis [Annatto $\mathrm{B} / \mathrm{C}$ (E 160b) and beta cyclodextrin (E 459)] and accumulation of eosinophilic material [beta-apo-8'-carotenal (E 160e)]. Nephrosis and nephrocalcinosis were identified as critical endpoints for several food additives, among which most structurally related and some only at the highest dose tested, including Ponceau 4R (E 124), sorbitan monostearate (E 491), sorbitan tristearate (E 492), sorbitan monolaurate (E 493), sorbitan monooleate (E 494) and sorbitan monopalmitate (E 495) for calcinosis, and phosphoric acid (E 338), sodium phosphates (E 339), potassium phosphates (E 340), calcium phosphates (E 341) and magnesium phosphates (E 343) for nephrocalcinosis. However, no AOPs are presently available for these specific adverse outcomes in the AOP Wiki.

Other AOPs that can be linked to critical toxic endpoints of food additives include:

- Testicular toxicity (AOP18/66/67/68/74/212) for cyclamate (E 952).

- Neurodevelopmental changes or toxicity (AOP8/12/13/1 7/42/48/54/134/152) for aluminium (E 173), aluminium sulphate (E 520), aluminium sodium sulphate (E 521), aluminium potassium sulphate (E 522), aluminium ammonium sulphate (E 523), sodium aluminium phosphate acid (E 541), glutamic acid (E 620), monosodium glutamate (E 621), monopotassium glutamate (E 622), calcium diglutamate (E 623), monoammonium glutamate (E 624) and magnesium diglutamate (E 625).

- Hematological changes and/or toxicity (AOP31) for ethyl lauroyl arginate (E 243), propyl gallate (E 310), tertiary-
Table 5 AOPs relevant to food additives available in the AOP Wiki

\begin{tabular}{ll}
\hline Toxicological endpoint & AOP number in AOP Wiki \\
\hline Hepatic steatosis and cholestasis & AOP27/34/36/57/58/59/60/61/62/232 \\
Nephrotoxicity & AOP33/105/116/128/138/177/186/256/257/258 \\
Testicular toxicity & AOP18/66/67/68/74/212 \\
Neurodevelopmental toxicity & AOP8/12/13/17/42/48/54/134/152 \\
Hematotoxicity & AOP31 \\
\hline
\end{tabular}


butyl hydroquinone (E 319), butylated hydroxytoluene (E 321), sorbitan monooleate (E 494), iron tartrate (E 534 ), hydrogenated poly-1-decene (E 907) and propane1,2-diol (E1520).

- Methemoglobin formation (KE in AOP31) for potassium nitrite (E 249), sodium nitrite (E 250), sodium nitrate (E 251), and potassium nitrate (E 252).

- Reduced hemoglobin levels (KE in AOP31) for azorubine (E 122) and stannous chloride (E512).

Only for two food additives, the critical toxic endpoint identified in the safety evaluation is carcinogenesis, namely dimethyl dicarbonate (E 242) (i.e., liver) and saccharins (E 954) (i.e., bladder). As previously mentioned, methyl carbonate [i.e., one of the reaction products of dimethyl dicarbonate (E 242)] was found to bioaccumulate in the liver, which leads to inflammation and hyperplasia, and ultimately to liver adenomas and carcinomas. This could be covered by AOP220. Other cancer-related AOPs in the AOP Wiki apply to other organs such as lung (AOP272), breast (AOP199/200), ovaries (AOP165), uterus (AOP167) and the hematopoietic system (AOP202). No AOPs related to bladder cancer were found.

It should be noted that for several food additives, such as iron tartrate (E 534), annatto, bixin, norbixin (E 160b), brown HT (E 155) and butylated hydroxytoluene (E 321), the safety assessment relied on a number of systemic adverse effects, involving different target organs. This means that different AOPs, which may or may not be mutually related, could be potentially used for the evaluation of a specific food additive.

For some food additives, the critical endpoints are local effects. For example, gastrointestinal irritation or corrosivity have been identified as the critical effects of acidic and alkaline food additives, including sulfur oxide (E 220), sodium sulphite (E 221), sodium bisulphite (E 222), sodium metabisulphite (E 223), potassium metabisulphite (E 224), calcium sulphite (E 226), calcium hydrogen sulphite (E 227), potassium bisulphite (E 228), hydrochloric acid (E 507) and sulphuric acid (E 513). Likewise, propionic acid (E 280), sodium propionate (E 281), calcium propionate (E 282) and potassium propionate (E 283) have been associated with local effects in the esophagus. AOPs for all these local endpoints are currently lacking.

\section{Discussion}

In the last 2 decades, a clear tendency has emerged to move to the use of mechanistic and $3 \mathrm{R}$ (i.e., replacement, refinement and reduction of animal studies)-based strategies for the safety evaluation of chemicals. This paradigm shift has been reinforced by the introduction of AOPs, which are gaining momentum worldwide. The application of AOPs in chemical risk assessment involves the possibility to predict apical toxic outcomes using molecular effects, which runs in parallel with the development and implementation of mechanistic approaches in toxicity testing (Willet et al. 2018). It should, however, be stressed that AOPs cannot be used as stand-alone tools, as they consider only the toxicodynamic basis of the adverse outcome. AOPs should therefore be combined with information on other critical aspects for sound safety evaluation, including exposure and kinetics as well as definitions of the critical effect and dose-response assessments. Together, a suitable PoD for risk assessment, such as a NOAEL or BMDL, may be established. AOPs have been proposed as frameworks to develop integrated testing strategies (Vinken et al. 2017). Such AOP-based integrated approaches to testing and assessment have been introduced and successfully applied in the cosmetics field, in particular for predicting and testing skin sensitization by identifying the possible hazards for this selected endpoint (OECD 2016). The AOP for the latter has been recently used as the basis for the establishment of a proposed AOP for allergic sensitization to food (Van Bilsen et al. 2017). There is high potential for the application of the AOP framework also in food chemical risk assessment. This has been illustrated by a number of recent publications, which show that PoDs derived from molecular endpoints, such as from toxicogenomic data and/or quantitative in vitro-to-in vivo extrapolation approaches, are often in the range of PoDs derived from traditional apical toxic endpoints (Levorato et al. 2019 and references therein). Driven by these recent developments, the task force of "New approaches to chemical risk assessment for foods and food ingredients" at ILSI Europe installed an expert group, entitled "Adverse outcome pathways and risk assessment", in 2017 with the aim of exploring the potential role of AOPs for contemporary safety evaluation in the food sector. This was achieved by first mapping the critical toxicological effects upon which the safety assessment of European-approved food additives is based. The expert group then mapped coverage of these adverse effects by AOPs publicly available in the AOP Wiki.

Safety assessment of food additives is currently performed according to the approach outlined by the WHO (2009). Of note, EFSA, in its guidance for submission of food additive evaluation, stressed the importance of adopting testing strategies in line with the $3 \mathrm{R}$ concept, also indicating the use of mode-of-action, and thus potentially of AOPs, in the process (EFSA 2012). This EFSA guidance indicates, for example, that use of mode-of-action is important to set a group ADI for compounds with, or presumed to have, a common mode-of-action. Another area where AOPs may be relevant in the safety evaluation of food additives is genotoxicity testing. The above-mentioned EFSA guidance states that insight into the mode-of-action, including, for instance, 
metabolic pathways involved in bioactivation, may be essential to define the in vitro assay appropriate for detection of possible genotoxicity. Modifications of the testing strategy are required when standard in vitro systems used for genotoxicity testing lack specific metabolic pathways or cannot mimic specific modes-of-action (EFSA 2012). This would hold for all cases where in vitro data are to be used in the process of safety evaluation. Clearly, the use of AOPs can support selection of the most appropriate in vitro systems.

In the guidance document, EFSA also mentions the use of the mode-of-action in a proposed tiered approach in which results of equivocal relevance in a preferred species may trigger use of such information in risk assessment. An example would be species-specific differences in the modeof-action underlying chemical-induced induction of kidney tumors only in male rats, but not in female rats or humans related to expression of alpha $2 \mathrm{u}$-globulin (Doi et al. 2007). Another example can be found in the hormone feedback loops in thyroid function in rats (Hard 2018), explaining why many substances that induce rodent thyroid tumors do not act as carcinogens in humans (Boobis et al. 2016). Thus, the use of including AOPs in safety assessment can be to support the human relevance of the effects observed in a test species. In the tiered approach for food additive evaluations proposed by EFSA (2012), mode-of-action studies may also be indicated in tier 3 when the results from regular OECDbased guideline studies in tier 2 trigger such additional studies, usually on a case-by-case basis. When describing the role of mode-of-action in the guidance document, the respective section indicates its use to support the relevance of findings in animals for humans. More specifically, it is mentioned that such studies can examine the mode-ofaction for carcinogenic effects or other endpoints, including endocrine disruption. EFSA also points out that these studies should use appropriate mode-of-action frameworks when assessing the data. This illustrates that in the currently applicable EFSA guidance for food additive evaluation, the AOP concept is included in the form of consideration of the mode-of-action, particularly for supporting selection of the correct in vitro system or animal model for the human situation. Taking this into account, one could foresee that including the AOP concept in the safety evaluation of food additives may also provide support for defining AOP-based definition of chemical-specific adjustment factors that deviate from the default uncertainty factors for interspecies or intraspecies differences in toxicodynamics.

The overview of critical effects used in the safety evaluation of food additives presented in this study provides a way forward to match the current use of the mode-of-action concept in safety evaluation of food additives and future AOP development. The data obtained revealed that often such detailed analysis of the mode-of-action would not be required because for $60 \%$ of the food additives, the EFSA/
SCF/JECFA safety evaluations did not identify a PoD for adverse effects. This probably reflects that, in contrast to other chemicals, such as pesticides, food additives are not designed to cause effects. For $22 \%$ of the food additives, the database for the established list of food additives listed in Annex II of Regulation (EC) No 1333/2008 (EC1333/2008), as summarized in Table S1 (supplementary information), identified specific critical adverse effects in animals. Replacement of the current default procedure for safety evaluation of food additives, also including 3R-alternative testing strategies, will require better use of insights into the modes-of-actions for both kinetic as well as dynamic characteristics. AOPs have the potential to provide insights into the toxicodynamics of a mode-of-action required to select the proper testing models not only for genotoxicity and carcinogenicity, as already done on a case-by-case basis, but also for other critical adverse effects. Further screening of the self-compiled database in Table S1 (supplementary information) of the present paper for relevant critical adverse effects food additives listed in Annex II of Regulation (EC) No 1333/2008 (EC1333/2008), in particular those used for establishing ADIs, showed that the most frequent critical toxic endpoints used for the safety assessment of food additives are related to liver and kidney effects. Other critical endpoints involved the cardiovascular system, the lymphatic system, the central nervous system and the reproductive system. AOPs are available for many of these apical endpoints in the AOP Wiki. However, they show different degrees of maturity, which could hamper their current use for safety evaluation purposes. For a number of other adverse outcomes pertinent to food additives, AOPs are completely lacking. This particularly holds true for irritation and/or corrosion of the different tissues in the gastrointestinal tract as well as for specific types of cancer, such as bladder cancer. Therefore, to further advance the use of AOPs in safety evaluation of food additives, efforts should be focused on developing AOPs for these particular toxicities. While doing so, and particularly in view of fit-for-purpose applications in the food additives field, quantification of these AOPs should be set as a priority. This can be achieved in a number of ways, such as by applying Bayesian computational methods, systems biology approaches and mathematical modeling of KE relationships employing machine learning. Furthermore, it should be kept in mind that individual AOPs are merely pragmatic tools for development and evaluation. For real-life applications, such as for the safety evaluation of food additives, so-called AOP networks should be used. AOP networks basically consist of combinations of individual AOPs with different MIEs and shared KEs (Knapen et al. 2018; Willett et al. 2018). AOP networks have already been proposed for a number of frequently occurring adversities, including liver steatosis (Mellor et al. 2016). It can be anticipated that upon development of such quantified AOP 
networks for critical effects relevant to the safety assessment of food additives as well as their implementation in integrative testing strategies, new avenues will be opened for innovative mechanistic safety evaluation approaches in the food industry, providing improved reliability while using fewer or no animals.

Acknowledgements This work was conducted by an expert group of the European branch of the International Life Sciences Institute (ILSI Europe). The experts received funding from the ILSI Europe task force "New approaches to chemical risk assessment for foods and food ingredients" to convene biannually. Industry members of this task force are listed on the ILSI Europe website at www.ilsi.eu. Experts are not paid for the time spent on this work, however, the non-industry members within the expert group were offered support for travel and accommodation costs to attend meetings for discussing the manuscript, and a small compensatory sum (honorarium) with the option to decline. The expert group carried out the work, i.e., collecting/analysing data/ information and writing the scientific paper separate to other activities of the task force. The opinions expressed and the conclusions of this publication are those of the authors and do not necessarily represent the views of ILSI Europe nor those of its member companies. The research reported is the result of a scientific evaluation in line with ILSI Europe's framework to provide a precompetitive setting for publicprivate partnership (PPP). ILSI Europe facilitated scientific meetings and coordinated the overall project management and administrative tasks relating to the completion of this work. For further information about ILSI Europe, please e-mail info@ilsieurope.be. The authors are grateful to current ILSI Europe staff member Mrs. Belinda Antonio as well as to former ILSI Europe staff members Dr. Bettina Schelkle and Dr. Kirsi Forsberg for their indispensable assistance in organizing all activities of the "Adverse outcome pathways and risk assessment" expert group at ILSI Europe. The authors also wish to thank the attendees of the ILSI Europe workshop "The use of AOPs for the safety evaluation of food additives" organized on 26-27 February 2019 in Brussels, Belgium for their valuable input on this manuscript.

Open Access This article is distributed under the terms of the Creative Commons Attribution 4.0 International License (http://creativeco mmons.org/licenses/by/4.0/), which permits unrestricted use, distribution, and reproduction in any medium, provided you give appropriate credit to the original author(s) and the source, provide a link to the Creative Commons license, and indicate if changes were made.

\section{References}

Ankley GT, Bennett RS, Erickson RJ et al (2010) Adverse outcome pathways: a conceptual framework to support ecotoxicology research and risk assessment. Environ Toxicol Chem 29:730-741

Becker RA, Ankley GT, Edwards SW et al (2015) Increasing scientific confidence in adverse outcome pathways: application of tailored Bradford-Hill considerations for evaluating weight of evidence. Regul Toxicol Pharmacol 72:514-537

Blaauboer BJ, Boobis AR, Bradford B et al (2016) Considering new methodologies in strategies for safety assessment of foods and food ingredients. Food Chem Toxicol 91:19-35

Boobis AR, Cohen SM, Dellarco VL et al (2016) Classification schemes for carcinogenicity based on hazard-identification have become outmoded and serve neither science nor society. Regul Toxicol Pharmacol 82:158-166
Burden N, Sewell F, Andersen ME et al (2015) Adverse outcome pathways can drive non-animal approaches for safety assessment. J Appl Toxicol 35:971-975

Dean JL, Zhao QJ, Lambert JC, Hawkins BS, Thomas RS, Wesselkamper SC (2017) Application of gene set enrichment analysis for identification of chemically induced, biologically relevant transcriptomic networks and potential utilization in human health risk assessment. Toxicol Sci 157:85-99

Delrue N, Sachana M, Sakuratani Y, Gourmelon A, Leinala E, Diderich R (2016) The adverse outcome pathway concept: a basis for developing regulatory decision-making tools. ATLA Altern Lab Anim 44:417-429

Doi AM, Hill G, Seely J, Hailey JR, Kissling G, Bucher JR (2007) $\alpha 2 \mathrm{u}-$ globulin nephropathy and renal tumors in National Toxicology Program studies. Toxicol Pathol 35:533-540

Edwards SW, Tan YM, Villeneuve DL, Meek ME, McQueen CA (2016) Adverse outcome pathways-organizing toxicological information to improve decision making. J Pharmacol Exp Ther 356:170-181

EFSA (2009) Existing approaches incorporating replacement, reduction and refinement of animal testing: applicability in food and feed risk assessment. EFSA J 7:1052

EFSA (2012) Guidance for submission of food additives evaluations. EFSA J 10:2760

EFSA (2014a) Statement on a conceptual framework for the risk assessment of certain food additives re-evaluated under Commission Regulation (EU) No 257/2010. EFSA J 12:3697

EFSA (2014b) Modern methodologies and tools for human hazard assessment of chemicals. EFSA J 12:3638

EFSA (2015) Scientific Opinion on the safety of the complexation product of sodium tartrate and iron(III) chloride as a food additive. EFSA J 13:3980

EFSA (2017) Re-evaluation of polyglycerol polyricinoleate (E 476) as a food additive. EFSA J 15:4743

EFSA (2018) Re-evaluation of oxidised soya bean oil interacted with mono- and diglycerides of fatty acids (E 479b) as a food additive. EFSA J 16:5420

Embry MR, Bachman AN, Bell DR et al (2014) Risk assessment in the 21st century: roadmap and matrix. Crit Rev Toxicol 44:6-16

Hard GC (2018) Mechanisms of rodent renal carcinogenesis revisited. Toxicol Pathol 46:956-969

Knapen D, Angrish MM, Fortin MC et al (2018) Adverse outcome pathway networks I: development and applications. Environ Toxicol Chem 37:1723-1733

Leist M, Ghallab A, Graepel R et al (2017) Adverse outcome pathways: opportunities, limitations and open questions. Arch Toxicol 91:3477-3505

Levorato S, Rietjens IMCM, Carmichael PL et al (2019) Novel approaches to derive points of departure for food chemical risk assessment. Curr Opin Food Sci. https://doi.org/10.1016/jcofs .2019 .02 .016 (in press)

Meigs L, Smirnova L, Rovida C, Leist M, Hartung T (2018) Animal testing and its alternatives: the most important omics is economics. Altex 35:275-305

Mellor CL, Steinmetz FP, Cronin MTD (2016) The identification of nuclear receptors associated with hepatic steatosis to develop and extend adverse outcome pathways. Crit Rev Toxicol 46:138-152

NRC (2007) Toxicity testing in the 21st century: a vision and a strategy. National Research Council, National Academies Press, Washington D.C

OECD (2016) Guidance Document on the Reporting of Defined Approaches and Individual Information Sources to be Used within Integrated Approaches to Testing and Assessment (IATA) for Skin Sensitisation. Series on Testing and Assessment No. 256:ENV/JM/MONO(2016)29 
OECD (2017) Revised guidance document on developing and assessing adverse outcome pathways. Series on Testing and Assessment 184:1-32

Perkins EJ, Antczak P, Burgoon L et al (2015) Adverse outcome pathways for regulatory applications: examination of four case studies with different degrees of completeness and scientific confidence. Toxicol Sci 148:14-25

Van Bilsen JHM, Sienkiewicz-Szłapka E, Lozano-Ojalvo D et al (2017) Application of the adverse outcome pathway (AOP) concept to structure the available in vivo and in vitro mechanistic data for allergic sensitization to food proteins. Clinical and Translational Allergy 7:13

Villeneuve DL, Crump D, Garcia-Reyero N et al (2014) Adverse outcome pathway (AOP) development I: strategies and principles. Toxicol Sci 142:312-320

Vinken M, Knapen D, Vergauwen L, Hengstler JG, Angrish M, Whelan M (2017) Adverse outcome pathways: a concise introduction for toxicologists. Arch Toxicol 91:3697-3707
WHO F (2009) International Programme on Chemical Safety (IPCS): Principles and methods for the risk assessment of chemicals in food. Environmental Health Criteria 240 ISBN: 978924157240 8

Willett C, Fitzpatrick S, Meek B, Westmoreland C (2018) Use of Adverse Outcome Pathways in Human Risk Assessment and Toxicology. In: Garcia-Reyero N, Murphy C (eds) A Systems Biology Approach to Advancing Adverse Outcome Pathways for Risk Assessment. Springer, Cham, https://doi.org/10.1007/9783-319-66084-4_17

Publisher's Note Springer Nature remains neutral with regard to jurisdictional claims in published maps and institutional affiliations. 